\title{
Background Noise Improves Gap Detection in Tonically Inhibited Inferior Colliculus Neurons
}

\author{
WILLARD W. WILSON AND JOSEPH P. WALTON \\ Department of Surgery, Division of Otolaryngology, University of Rochester School of Medicine and Dentistry, Rochester, \\ New York 14642
}

Received 14 March 2001; accepted in final form 4 October 2001

Wilson, Willard W. and Joseph P. Walton. Background noise improves gap detection in tonically inhibited inferior colliculus neurons. J Neurophysiol 87: 240-249, 2002; 10.1152/jn.00218.2001. Single units in the inferior colliculus (IC) in the C57B1/6 inbred mouse strain were tested for their temporal processing ability as measured by their minimum gap threshold (MGT), the shortest silent interval in an ongoing white-noise stimulus which a unit could encode. After ascertaining the MGT in quiet, units were re-tested in various levels of background noise. The focus of this report is on two types of tonically responding units found in the IC. Tonically inhibited (TI) units encoded gaps poorly in quiet and low levels of background noise as compared with tonically excited (TE) units. In quiet, the MGTs of TI units were about an order of magnitude longer than the MGTs typical of TE units. Paradoxically, gap encoding was improved in high levels of background noise for TI units. This result is unexpected from the traditional viewpoint that noise necessarily degrades signal processing and is inconsistent with psychophysical observations of diminished speech and gap detection processing in noisy environments. We believe the improved feature detection described here is produced by the adaptation of inhibitory input. Continuous background noise would diminish the inhibitory efficacy of the gap stimulus by increasing the latency to the onset of inhibition and decreasing its duration. This would allow more spontaneous activity to "bleed through" the silent gap, thus signaling its presence. Improved feature detection in background noise resulting from inhibitory adaptation would seem an efficient neural mechanism and one that might be generally useful in other signal detection tasks.

\section{N T R O D U C T I O N}

Human speech and other species-specific communication signals are characterized by rapid intensity fluctuations, which are thought to be important information-bearing parameters for signal processing (Rosen 1992). Such rapid intensity changes can be modeled using brief silent intervals, or "gaps" in an ongoing stimulus. Behavioral gap detection paradigms are reliable indicators of auditory temporal acuity (Fitzgibbons 1983; Glasberg et al. 1987; Green and Forrest 1989; Plomp 1964; Shailer and Moore 1983) and gap detection correlates well with other, more complex processing, such as speech perception in noise (Glasberg and Moore 1988; Gordon-Salant and Fitzgibbons 1993; Tyler et al. 1982). In humans, minimal detectable gap durations are approximately 2-3 ms (Fitzgibbons and Wightman 1982; Green and Forrest 1989; Plomp 1964). Sim-

Present address and address for reprint requests: W. W. Wilson, TuckerDavis Technologies, 4637 NW 6th St., Gainesville, FL 32609 (E-mail: wwilson@tdt.com). ilar behavioral gap thresholds have been found in other species (Giraudi et al. 1980; Ison et al. 1993; Klump and Maier 1989; Okanoya and Dooling 1990; Walton et al. 1997).

Background noise typically elevates thresholds and impairs perception of complex signals. For example, gap detection thresholds are increased by filling the silent interval in a gap detection stimulus with noise (Forrest and Green 1987; Green and Forrest 1989). Similarly, speech discrimination is progressively impaired with increasing background noise level (Dubno et al. 1984; Stuart and Phillips 1996). This detrimental influence of background noise on speech processing is particularly pronounced in elderly listeners (Dubno et al. 1984; GordonSalant and Fitzgibbons 1993; Stuart and Phillips 1996). Our study was carried out to search for neurophysiological correlates to degraded temporal processing in background noise, using the gap detection paradigm.

In auditory nerve fibers, neural responses to gap detection stimuli exhibit diminished firing rates during the stimulus gap (Feng et al. 1994; Klump and Gleich 1991; Zhang et al. 1990). Minimal gap thresholds (MGTs) of auditory nerve fibers in chinchilla (Zhang et al. 1990) and starling (Klump and Gleich 1991) approximate psychophysical thresholds in the same species. Likewise, the shortest cortical MGTs in starling cortex (Buchfellner et al. 1989) were comparable to behavioral estimates of starling gap detection (Klump and Maier 1989). Walton et al. (1997) demonstrated that MGTs of mouse inferior colliculus (IC) neurons were comparable to behavioral estimates of gap detection in the same subjects. Similar to Buchfellner et al. (1989), they reported that phasic neurons, especially the onset class, had the shortest MGTs and that neural recovery with increasing gap duration mirrored behavioral startle inhibition.

We tested the neural response to gaps in the IC of the mouse. In the IC, inhibitory input is central in shaping the response latency (Park and Pollak 1993), intensity coding (Faingold et al. 1991), monaural frequency tuning (Yang et al. 1992), binaural response properties (Kuwada et al. 1997; Li and Kelly 1992), and spatial receptive fields (Park and Pollak 1994). Moreover, inhibition plays a pivotal role in higher order temporal processing such as duration tuning (Casseday et al. 1994; Ehrlich et al. 1997), AM selectivity (Palombi and Caspary 1996), FM selectivity (Fuzessery and Hall 1996), and delay

\footnotetext{
The costs of publication of this article were defrayed in part by the payment of page charges. The article must therefore be hereby marked "advertisement" in accordance with 18 U.S.C. Section 1734 solely to indicate this fact.
} 
tuning (Park and Pollak 1993; Saitoh and Suga 1995). Given the important role of inhibition in encoding the time course of auditory stimuli, neural inhibition might also be expected to be crucial to encoding gap duration in mouse IC neurons.

We explored the neural basis of degraded temporal processing in background noise by measuring the ability of IC neurons to encode the presence of a stimulus gap in quiet and various levels of background noise. Here, we report on a unique set of auditory neurons for which adding background noise actually improves their ability to encode acoustic features. Moreover, the data suggest that adaptation of the inhibitory input to these cells creates this enhanced performance in noisy conditions.

\section{METHODS}

A total of 19 mice of the $\mathrm{C} 57 \mathrm{Bl} / 6$ inbred strain were prepared for electrophysiological recording using methods described elsewhere (Walton et al. 1997). Briefly, the animal was anesthetized and a small threaded holding tube and an indifferent electrode were attached to the skull with cyanoacrylate adhesive and dental acrylic. A small hole (approximately $500 \mu \mathrm{M}$ diameter) was then drilled over the surface of the IC with a dental drill.

Electrophysiological recording was carried out in a soundproofed booth lined with acoustical foam. During experiments, animals were mildly tranquilized with Taractan $(5-12 \mathrm{~g} / \mathrm{kg})$. The head was stabilized using the holding tube, and borosilicate glass microelectrodes $(8-12 \mathrm{M} \Omega$ ) were advanced through the IC using a remote-controlled positioner. Single units were isolated using conventional electrophysiological techniques and verified to be in the central nucleus of the IC using horseradish peroxidase histochemistry (after Mesulam 1982). All surgical and recording procedures were approved under the animal care and usage guidelines of the University Committee on Animal Resources.

Acoustic stimuli were generated using a commercial digital signal processing platform (Tucker-Davis Technologies). The "foreground" gap stimuli were digitally generated noise bursts with a bandwidth of 2-60 kHz. Gap stimuli were generated using a D/A converter, attenuated, amplified, and sent to a high-frequency transducer (Panasonic model 100) placed $30^{\circ}$ contralateral to the recording site. A second D/A channel synthesized background noise, which was inversely gated around the foreground stimulus (ON when the foreground stimulus was OFF), attenuated and mixed with the gap stimuli before amplification. The frequency response of the system was normalized within $\pm 2 \mathrm{~dB}$ from $2-60 \mathrm{kHz}$.

Search stimuli were 100-ms-long noise or tone bursts (5-ms rise/fall time) presented at a rate of $4 / \mathrm{s}$. After isolation of a single unit, rate/intensity functions were gathered using 100-ms-long noise bursts, presented for 50 repetitions at each intensity. Functions were typically run at 5 - $\mathrm{dB}$ intervals from below threshold to $\geq 65 \mathrm{~dB}$ sound pressure level (SPL).

Automated "gap series" were then presented in quiet and in several different levels of background noise. Gap stimuli were $65 \mathrm{~dB}$ SPL noise bursts, with an overall duration of $150 \mathrm{~ms}$ and a 5-ms cosineshaped rise/fall time. A quiet gap of variable duration and $0.5-\mathrm{ms}$ rise/fall time was inserted into the noise burst, separating the first 100 $\mathrm{ms}$ of the gap stimulus (NB1) from the second $50 \mathrm{~ms}$ (NB2). The standard gap series included gaps of $1,3,6,12,24,48$, and $96 \mathrm{~ms}$ in addition to a 0-ms gap (control) stimulus. Additional gap durations were commonly run to obtain a more precise determination of the MGT within the standard gap series. Each member of a gap series was presented for 75 repetitions at a rate of $2 / \mathrm{s}$.

Our analysis focused on whether the neural response of a unit might encode the presence of a stimulus gap. We used a statistical comparison between the control response and each of the other (gap $>0-\mathrm{ms}$ ) responses to determine the MGT: the shortest gap duration producing a distinct and statistically significant response as compared with the control. Our window-based analysis was similar to that used in other studies (e.g., Barsz et al. 1998; Buchfellner et al. 1989; Feng et al. 1994; Walton et al. 1997, 1998) except that our analysis windows were tailored individually for each gap series, accounting for differences in response latency across units and stimulus configurations.

The first of these windows, the "gap window," corresponded to the interval between NB1 and NB2, after correction for the unit's temporal response pattern. The gap window started at the end of NB1 response and had a variable duration equal to the gap duration being tested. The bin containing the end of the NB1 response was subjectively determined from a composite of all of the PST histograms in a gap series added together. Gap windows longer than the control response were truncated to the end of the control response to exclude nondriven activity in the control PST histogram from entering the comparison.

The second analysis window, the "NB2 window" bracketed the response to NB2. The bins containing the start and end of the NB2 response were determined from a second composite histogram. This composite was constructed by shifting each gap peristimulus time (PST) histogram in the series "back in time" (to the left) by its gap duration before the addition, lining up all of the NB2 responses in the composite PST histogram. The start and end of the NB2 response was defined relative to the onset of NB2 in the stimulus. Thus, the NB2 window "followed" the response to NB2 at different gap durations.

The 75 spike counts (one for each of 75 stimulus presentations) in a given window were used as a distribution for statistical testing. The distributions for each gap duration were compared with the distributions in corresponding analysis windows in the control using a Wilcoxon signed-rank test. The MGT was defined as the shortest gap duration producing a significant difference in gap window or the NB2 window from the control at a significance level of $P \leq 0.05$, with the additional restriction that the next largest gap duration we tested also produced a significant effect.

The data reported here are part of a larger data set exploring the neural correlates of presbycusis. The C57Bl/6 inbred strain was selected for these experiments as an animal model for age-related hearing loss. However, the effects we report below were observed in both young and middle-aged individuals.

\section{R E S U L T S}

Gap series were obtained for 154 units across 19 animals in this study. Units first were characterized by their temporal response pattern to the control (0-ms gap) stimulus and we focus below on the 36 units exhibiting purely tonic responses. A tonically inhibited (TI) response pattern was observed in $6.5 \%$ of all units characterized with gap series $(n=10)$. TI responses are rare in other studies of the mouse IC (Walton et al. 1997, 1998; Willott et al. 1988) and within the central auditory system of other species (e.g., Buchfellner et al. 1989; Volkov and Galazjuk 1991). However, we found a unique response in TI units to the gap detection paradigm and below, we compare this response type to units with the mirror image response pattern, "sustained" or tonically excited (TE) units $(n=26)$.

Representative rate/intensity functions for these two unit types are contrasted in Fig. 1. TI units (left column, Fig. 1A) were characterized by a high level of spontaneous activity that was blocked by suprathreshold acoustic stimulation. The spontaneous firing rate measured from control PST histograms just before stimulus onset averaged 34 spikes/s across all TI units. The rate/intensity function for the TI unit shown in Fig. $1 B$ shows a monotonic increase in the inhibition of spontaneous activity with stimulus intensity, illustrated by the decreasing 


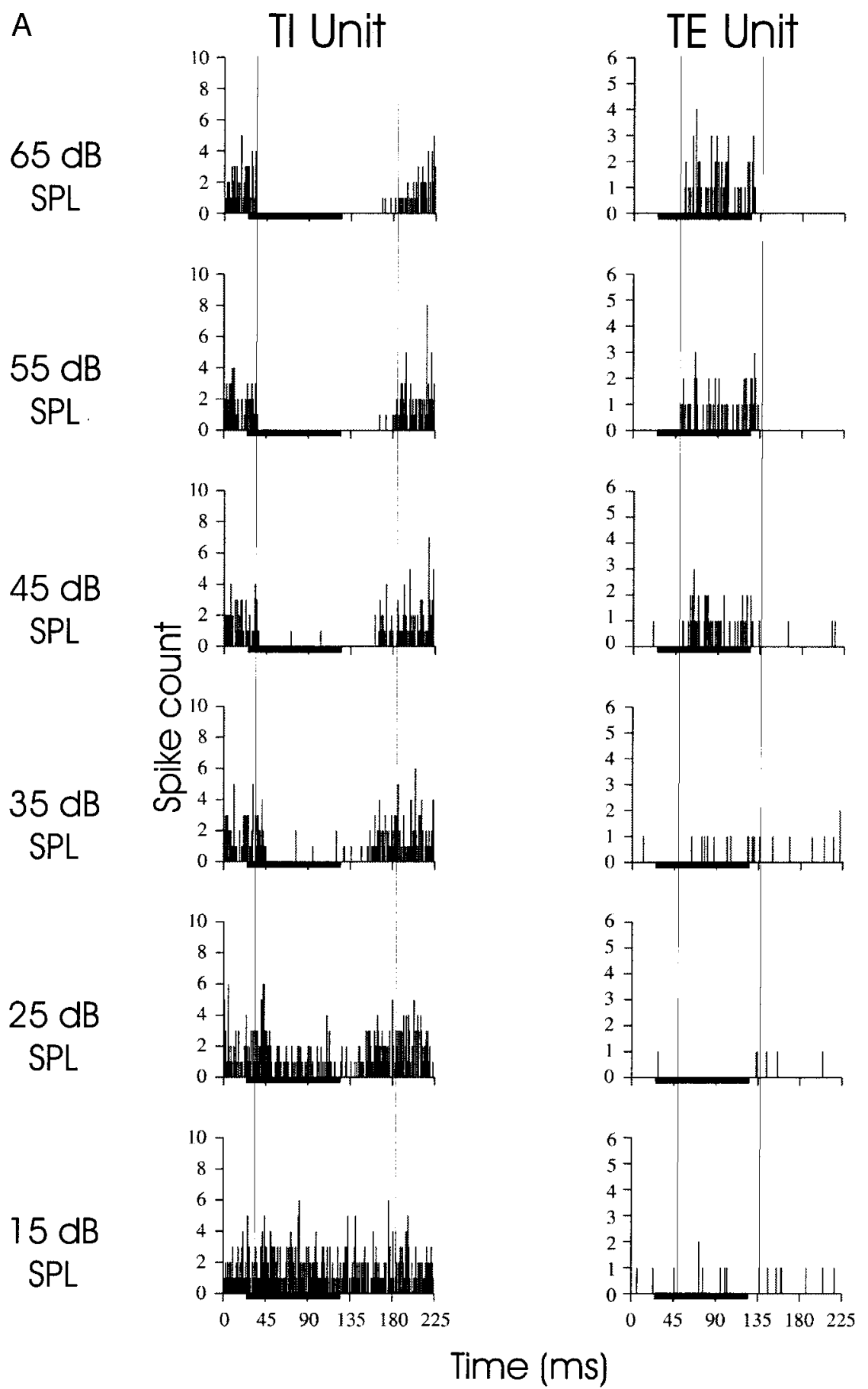

B

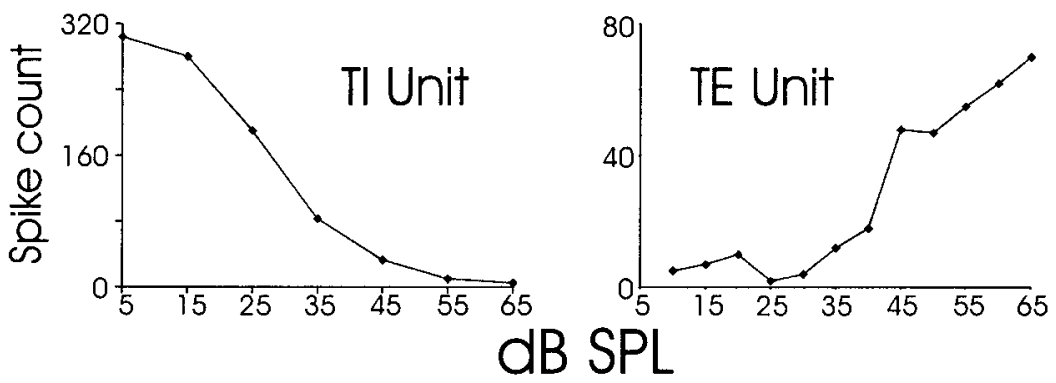

FIG. 1. A: temporal response patterns of a representative tonically inhibited (TI) unit (left) and tonically excited (TE) unit (right) to 100-ms noise bursts shown as a function of noise intensity. Intensities ranged from 15 to $65 \mathrm{~dB}$ sound pressure level (SPL) and 25 stimulus repetitions were presented. The dashed vertical lines show the spike count window for each of the functions determined from a composite histogram of all peristimulus time (PST) histograms combined. $B$ : corresponding rate/intensity functions for each unit. 
spike counts during acoustic stimulation. A similar monotonic inhibitory response was observed in most TI units, although three had nonmonotonic rate/intensity functions. These units had spike counts that were inhibited by $\geq 25 \%$ at intensities both higher and lower than that exhibiting the maximum spike count. Across all TI units, the firing rate at $65 \mathrm{~dB}$ SPL (the intensity of the gap stimulus) was decreased to an average of $11 \%$ of the maximum firing rate measured in rate/intensity functions.

In contrast, TE units (right column, Fig. 1A) generally had lower spontaneous firing rates (mean $=12 \mathrm{spikes} / \mathrm{s}$ ) and responded at a relatively constant rate throughout the duration of acoustic stimuli above threshold. In this example, the response magnitude increased monotonically with intensity (Fig. 1B). The average response for all TE units measured at $65 \mathrm{~dB}$ SPL was $70 \%$ of the maximum response obtained in the rate/ intensity functions. Monotonic responses $(<25 \%$ decrease in response at intensities greater than that eliciting the maximum response) were observed in $62 \%$ of TE units.

Typical responses of these two unit types to gap stimuli are shown in Fig. 2. Both tonic response types encoded stimulus gaps by a cessation of their (either excitatory or inhibitory) response during the gap. During NB1 and NB2, the inhibitory input to TI units eliminated the spontaneous activity. However, during gaps above the MGT, the inhibitory input was inactive and the spontaneous activity bled through during the silent interval. TE units responded at a steady rate throughout NB1, ceased responding during gaps above the MGT, and exhibited renewed, sometimes enhanced, excitation to NB2.

Based on their mirror-image response patterns, one might expect similar temporal acuity of TI and TE units in the gap detection paradigm. However, differences in gap-encoding capability between TI and TE units were readily apparent (Fig. 3). The MGTs in TE units measured in quiet ranged from 1 to $12 \mathrm{~ms}$ (mean $=2.9$ ), typical of other unit types displaying an excitatory response pattern (data not shown). However, the MGTs in TI units were about an order of magnitude higher than the TE units, ranging from 12 to $96 \mathrm{~ms}$ (mean $=52.2$ ).

Figure 4 shows four standard gap series for a single TI neuron. Each column shows the response in different background noise levels, with asterisks indicating the shortest gap duration producing a significantly different response from the

\section{TI Unit}

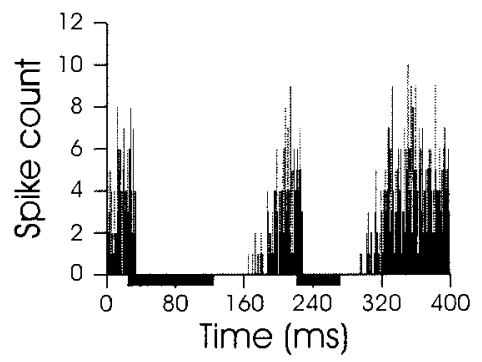

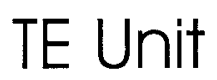

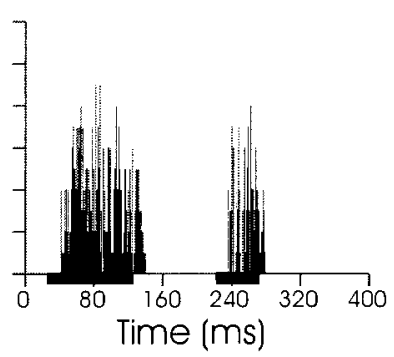

FIG. 2. Discharge patterns to a gap stimulus of $96 \mathrm{~ms}$ by a TI unit and a TE unit. Note that the discharge patterns are mirror images of one another. That is, the TE unit discharges (right) only during Noise Burst 1, first $100 \mathrm{~ms}$ of gap stimulus (NB1) and Noise Burst 2, last $50 \mathrm{~ms}$ of gap stimulus (NB2). In contrast, the TI unit (left) ceases discharging during NB1 and NB2. There is, however, a slow increase in the number of discharges during the gap window, signaling the presence of the gap.

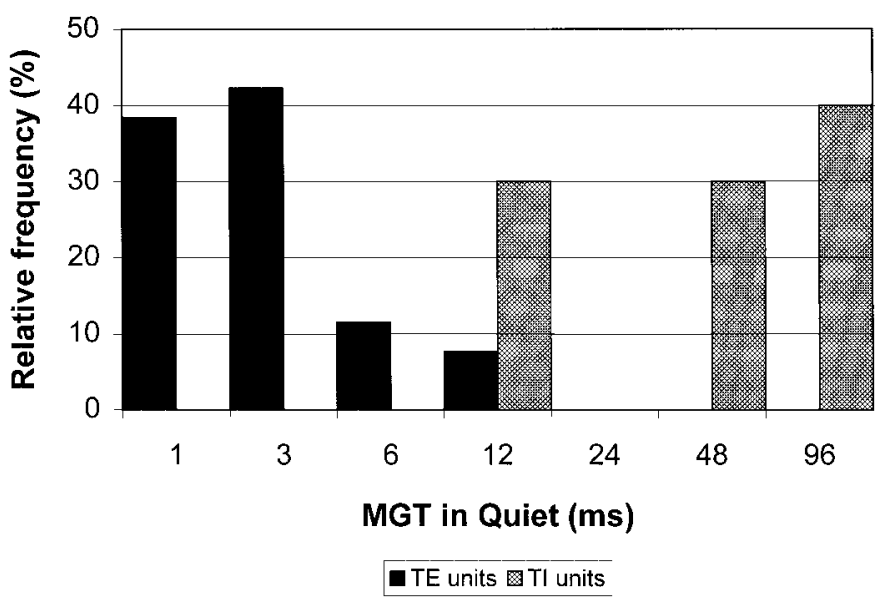

FIG. 3. Frequency distribution of minimum gap thresholds (MGTs) in quiet for the TI (light bars) and TE (dark bars) unit types. Note that none of the TI units had gap thresholds below $12 \mathrm{~ms}$ in quiet, while all of the TE units had MGTs $\leq 12 \mathrm{~ms}$.

control. In the absence of background noise (leftmost column), silent gaps $\leq 48 \mathrm{~ms}$ long did not elicit a gap response. Only when the gap duration was increased to $96 \mathrm{~ms}$ did the firing rate during the silent interval between NB1 and NB2 become significantly different from the same interval in the control response $(P \leq 0.01)$. However, in contrast to the negative influence on signal processing traditionally attributed to background noise, gap detection in this unit was actually improved when a low-level continuous background noise, $36 \mathrm{~dB}$ below the level of the gap stimulus, was present (Fig. 4, 2nd column). Here, a response to a 48-ms-long gap was seen in addition to the response to the 96-ms gap observed in quiet. Further increasing the level of background noise to 24 and $12 \mathrm{~dB}$ below the gap carrier level systematically improved the ability of the neuron to encode short silent intervals (to 24 and $12 \mathrm{~ms}$, respectively; $3 r d$ and 4th columns). Across all TI units and background noise levels, background noise caused a decrease in MGT by an average of $24 \mathrm{~ms}$, as compared with quiet.

The response of a typical TE is shown in Fig. 5. In quiet (left column), a statistically significant decrement in the response during the gap window was first observed at a 3-ms-gap duration $(P \leq 0.01)$ and was present at all longer gap durations tested. In addition, the response to the onset of NB2 was enhanced at gap durations longer than the MGT $(6,12,24$, and $48 \mathrm{~ms})$. In background noise, the gap detection capability of this TE unit was unaltered (rightmost three columns); although raising the background noise level did increase the firing rate of the unit, particularly at $12 \mathrm{~dB} \mathrm{~S} / \mathrm{N}$. Background noise generally had little effect on the MGTs of TE units. MGTs were slightly increased by background noise, by an average of about $1.0 \mathrm{~ms}$ across all noise levels, as compared with quiet.

\section{Temporal analysis}

Given the large difference in the temporal acuity in quiet between TI and TE units in the gap detection paradigm, we thought that differences in the time course of their responses might also be observed to simpler stimuli. Figure 6 shows the distribution of neural response duration to 100 -ms noise bursts for the 36 tonic responders included in this study. An obvious difference between TI and TE units can be seen. The mean 


\section{Tl unit gap detection}

Gap Quiet $\quad 36 \mathrm{~dB}$ S/N $\quad 24 \mathrm{~dB}$ S/N $\quad 12 \mathrm{~dB}$ S/N

$\mathrm{O} \mathrm{ms}$

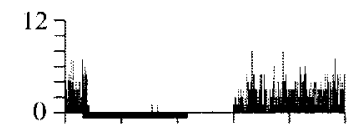

$1 \mathrm{~ms}$

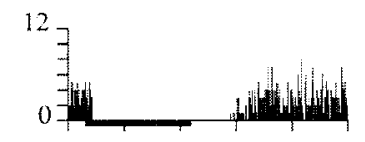

$3 \mathrm{~ms}$

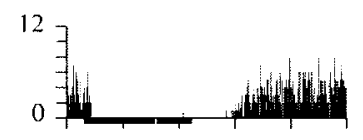

$6 \mathrm{~ms}$
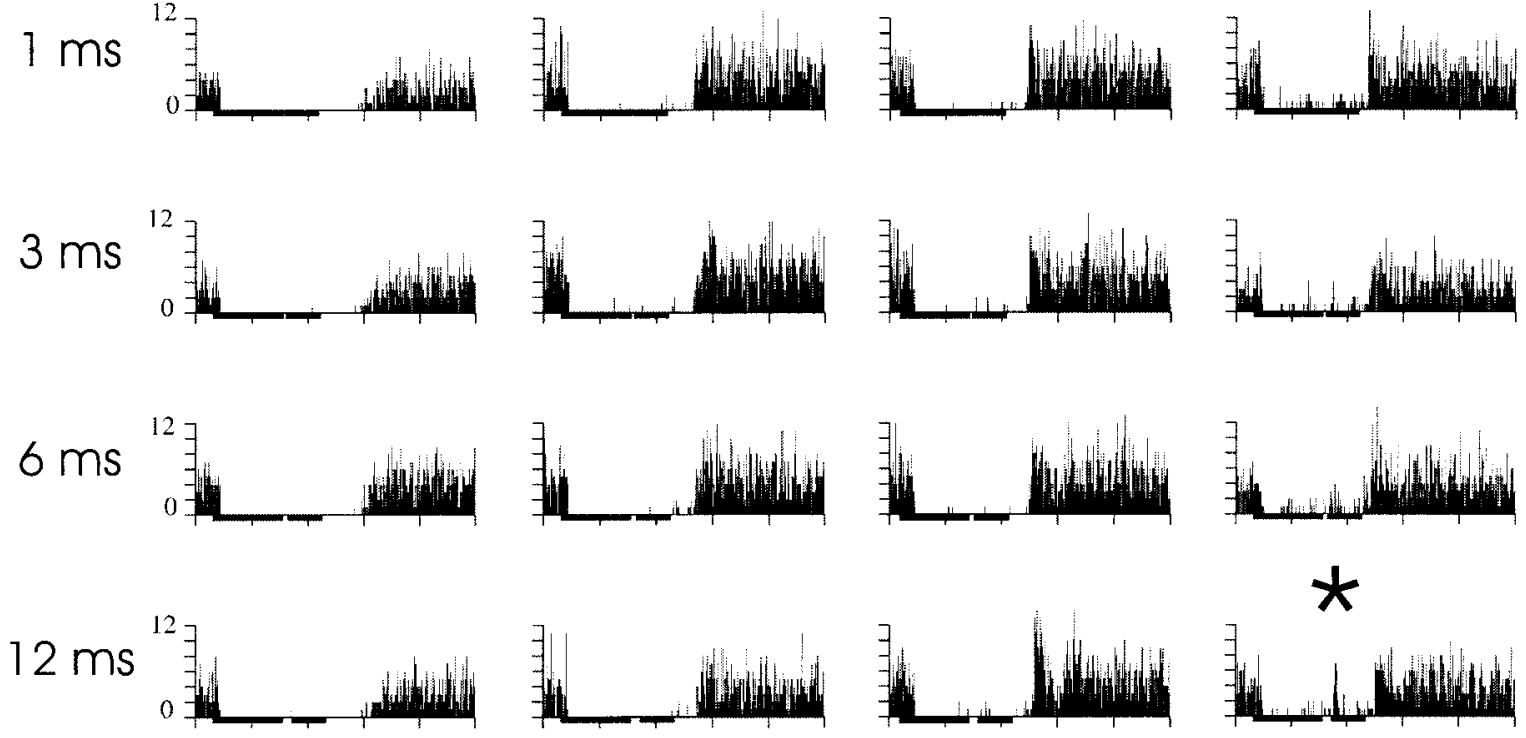

*
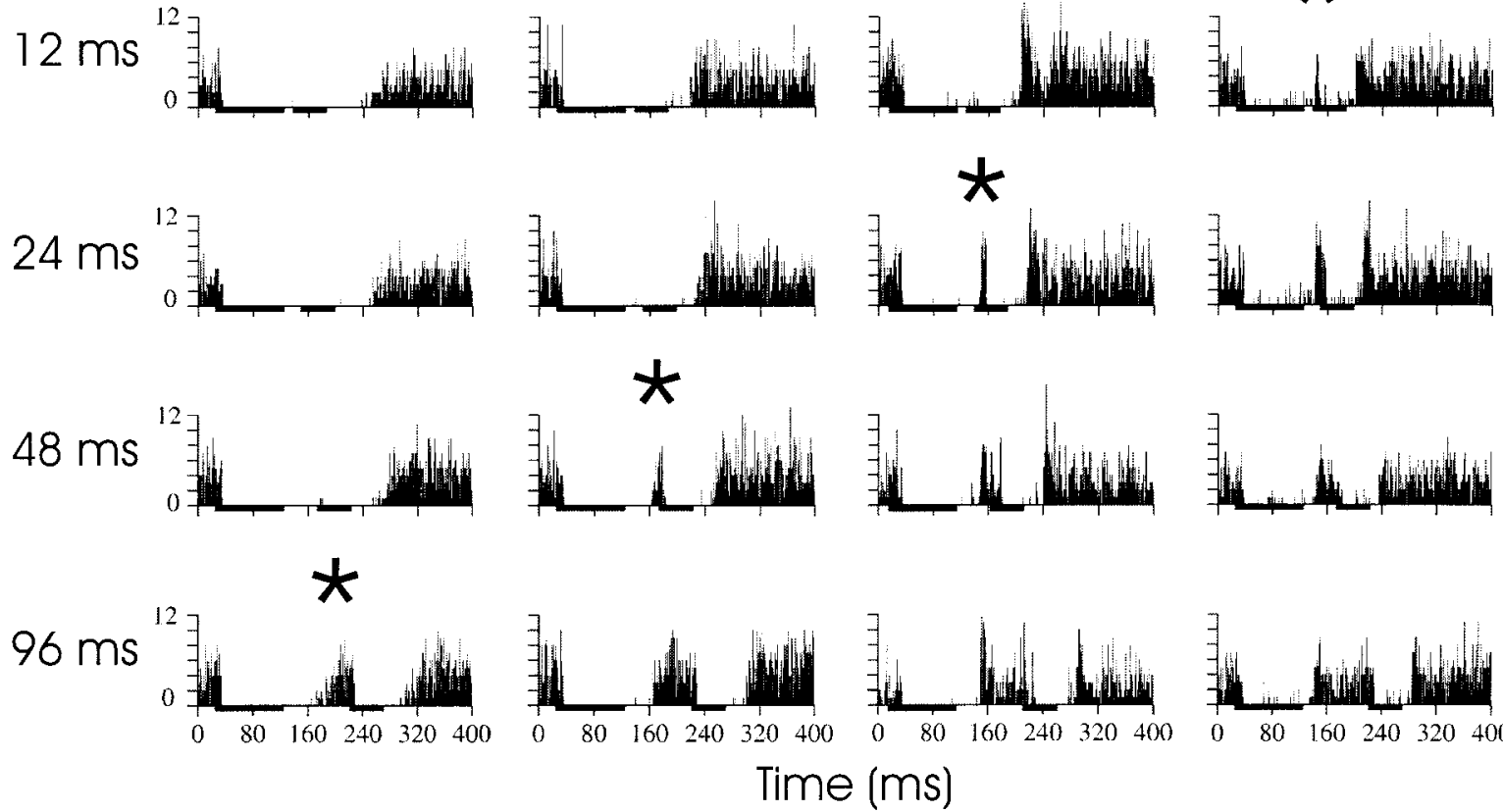

Time (ms)

FIG. 4. PST histograms collected from the standard gap series of a TI unit shown for four different S/N conditions (quiet, 36, 24 , and $12 \mathrm{~dB} \mathrm{~S} / \mathrm{N})$. The ambient room noise was $14 \mathrm{~dB}$ SPL, placing the S/N level of the gap stimulus in quiet at $>50 \mathrm{~dB}$ SPL. Asterisks denote the gap duration where a statistically significant difference from the control PST histogram was obtained. As the $\mathrm{S} / \mathrm{N}$ levels were increased from quiet to $12 \mathrm{~dB}$, the MGT decreased. All PST histograms were computed from 75 repetitions and the carrier intensity was $65 \mathrm{~dB}$ SPL.

response duration of the TI units was $148 \mathrm{~ms}(\mathrm{SD}=26.3)$ in response to 100-ms noise bursts and the response outlasted the stimulus in each of the TI units we measured. In contrast, the duration of TE responses averaged $91 \mathrm{~ms}(\mathrm{SD}=16.8)$ in response to $100-\mathrm{ms}$ noise bursts. Only $35 \%$ of the TE units had response durations longer than the stimulus.
Hence, in quiet, the temporal acuity of both TI and TE responders in the gap detection paradigm corresponds with their response duration. However, we observed that TI response duration can change dramatically with stimulus conditions. This can be seen in the rate/intensity function shown in Fig. 1, in which increasing the intensity of acoustic stimulation 


\section{TE unit gap detection}

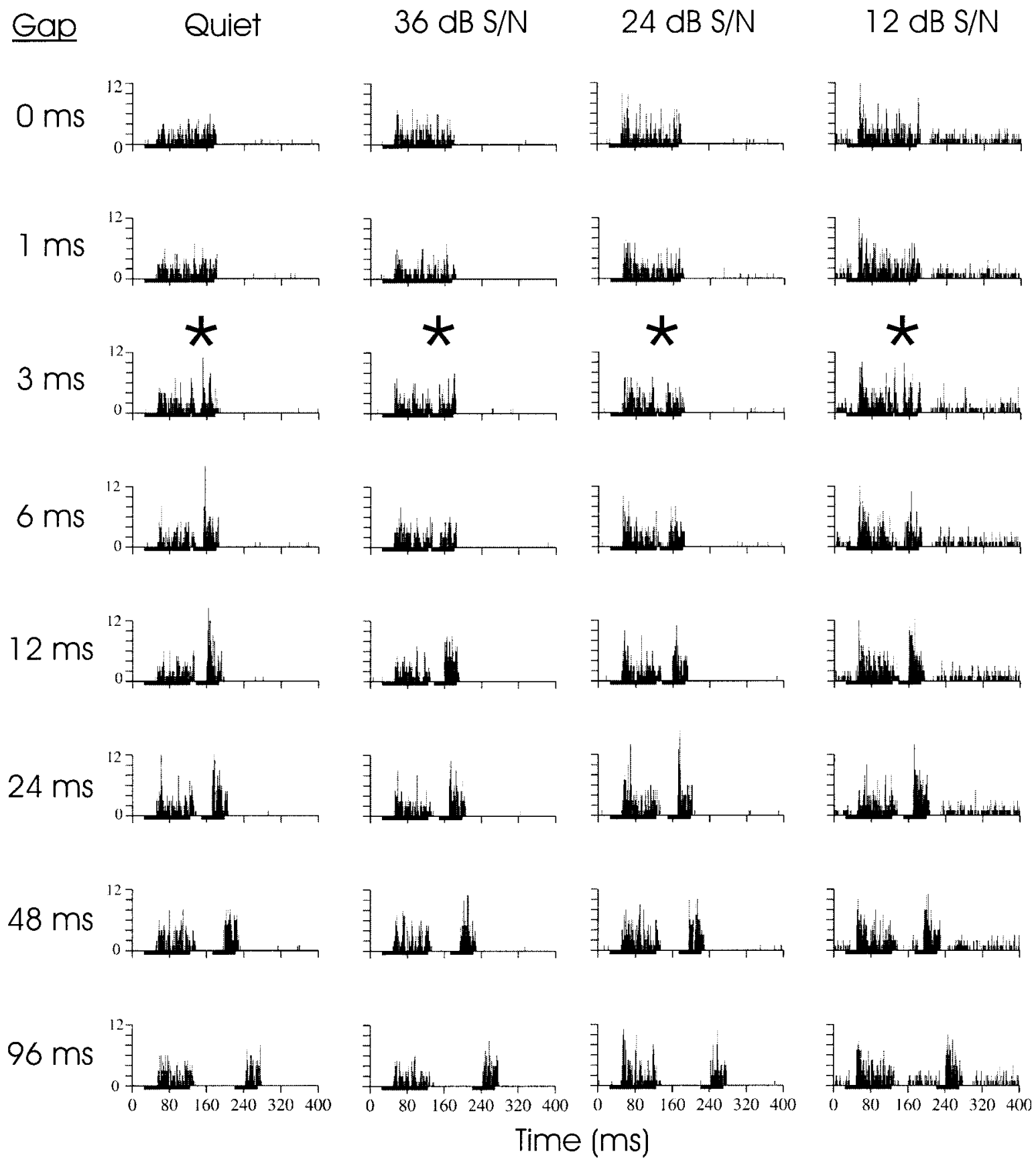

FIG. 5. Same format as Fig. 4, but for a TE unit. Asterisks denote the MGT for each series. Note that unlike the TI unit, as the $\mathrm{S} / \mathrm{N}$ level increased, MGT remained constant at $3 \mathrm{~ms}$.

not only diminished spike counts, but also increased the duration of the inhibitory response. A second factor having a profound influence on TI unit response duration was background noise level. For example, the control PST histograms of the TI unit in Fig. 4 (top row) show an obvious decrease in inhibitory duration at higher background noise levels. In quiet, the inhibition ended about $240 \mathrm{~ms}$ into the control PST histogram. In the $12 \mathrm{~dB}$ S/N condition, the same $65 \mathrm{~dB}$ SPL white noise burst produced an inhibitory response which ended about 50 ms sooner.
Diminished inhibitory duration in background noise was common in TI units. Figure 7 shows the influence of background noise on response duration to the control for two TI and two TE units. Both TI units (top) show marked decreases in response duration with background noise. In addition, a corresponding decrease in the MGTs measured at the same noise levels was observed in these units. The response duration in TE units did not exhibit such dramatic and systematic changes in response duration with background noise level (bottom), and MGTs remained relatively constant in background noise. 


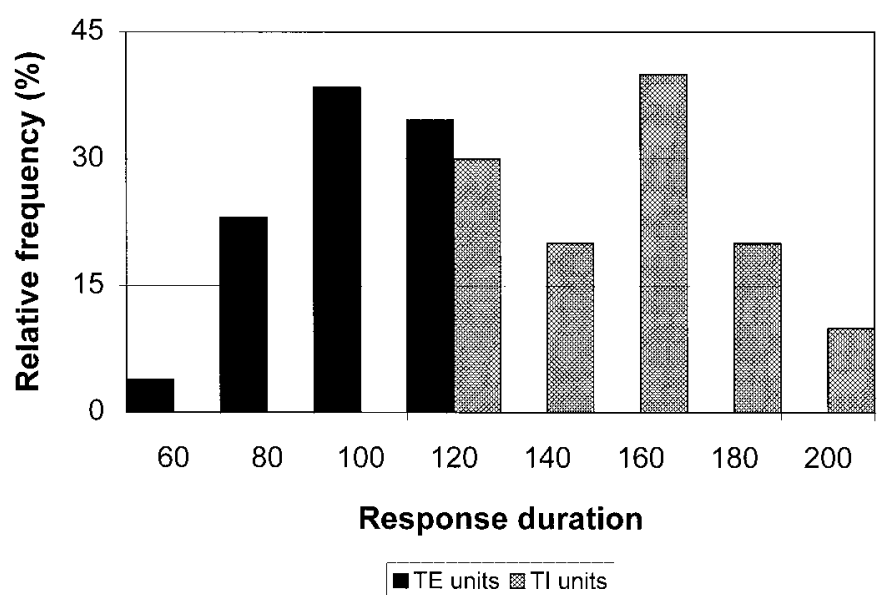

FIG. 6. Distribution of response durations across all TE (dark bars) and TI units (light bars) included in this study. To measure response duration, a composite histogram was computed from all PST histograms in the unit's rate/intensity function. Response onset and offset were subjectively determined from the composite histogram displayed using a 1-ms binwidth.

\section{I S C U S S I O N}

Counter to the conventional view that background noise must be detrimental to signal processing, we observed that gap encoding in tonically inhibited units was actually improved by adding background noise. Moreover, the degree to which MGTs improved was directly related to the $\mathrm{S} / \mathrm{N}$ ratio: $\mathrm{TI}$ units were capable of increasingly more sensitive temporal processing in increasingly unfavorable $\mathrm{S} / \mathrm{N}$ conditions. While pure tonic inhibition is rare in the IC (Walton et al. 1998, 1997; Willott and Urban 1978), inhibitory input is observed in the vast majority of IC cells (Klug et al. 1999) and plays a very important role in complex signal processing (e.g., Casseday et al. 1994; Fuzessery and Hall 1996; Kuwada et al. 1997; Palombi and Caspary 1996; Park and Pollak 1994). Given the above, our results that temporal processing by IC units with inhibitory input is improved by background noise prompts the question as to the role of inhibitory input in other complex signal processing tasks under adverse listening conditions.

In the following section, we discuss inhibitory response duration in relation to the temporal acuity of TI units. We then present a model for improved gap detection in background noise by TI units and discuss adaptation as a potential mechanism for generating paradoxical gap detection thresholds in background noise. Finally, we explore the potential influence of changing inhibitory duration on the detection of other acoustic features.

\section{Long inhibitory duration and poor temporal acuity in quiet}

Units with tonic inhibitory input generally exhibit the longest MGTs in the central auditory system (Buchfellner et al. 1989; Walton et al. 1997). Our data suggest that this poor temporal acuity in TI neurons is related to the duration of their inhibitory response. Since the stimulus gap can only be encoded only after the unit has stopped responding to NB1, a longer response to NB1 would shorten (or eliminate) the uninhibited period of spontaneous activity during the gap between NB1 and NB2 and diminish the temporal acuity of the cell.

In quiet, we observed that TI units did not accurately encode stimulus duration by their response duration. Rather, response duration exceeded the stimulus duration in each of the TI units and could be almost twice as long as the

\section{TI Units}

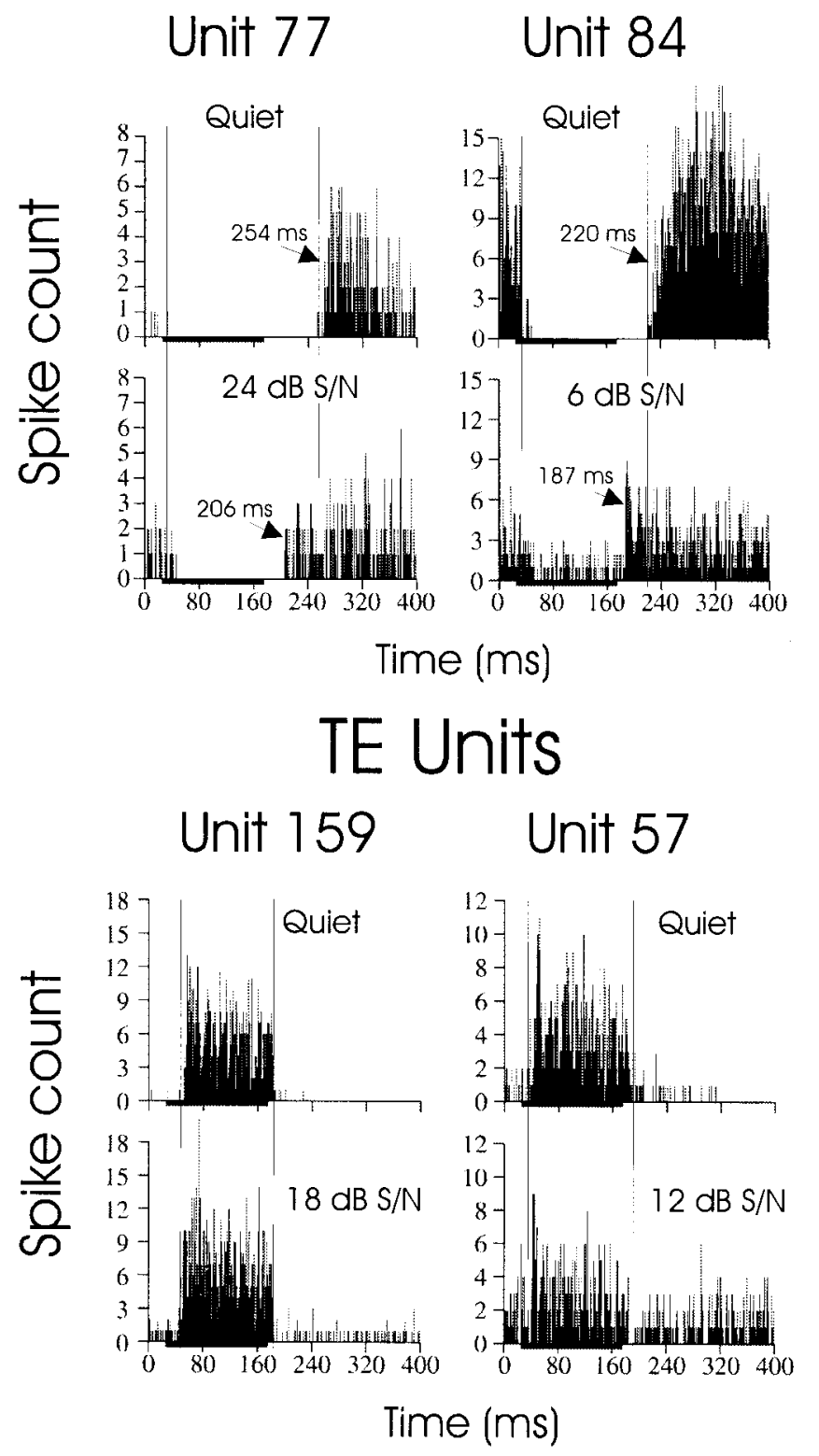

FIG. 7. PST histograms for two TI units (units 77 and 84) and two TE units (units 159 and 57) in quiet and background noise. Both TI units show marked decreases in response duration in background noise. For unit 77, the 150-ms control stimulus evoked inhibition which ended $254 \mathrm{~ms}$ into the PST histogram. The offset of inhibition was decreased to $206 \mathrm{~ms}$ in $24 \mathrm{~dB} \mathrm{~S} / \mathrm{N}$. Correspondingly, the MGT for this unit decreased from $96 \mathrm{~ms}$ in quiet to 18 $\mathrm{ms}$ in $24 \mathrm{~dB}$ S/N. The offset of inhibition in TI unit 84 was shortened by 33 $\mathrm{ms}$ in background noise and its MGT decreased from $48 \mathrm{~ms}$ in quiet to $6 \mathrm{~ms}$ at $6 \mathrm{~dB}$ S/N. TE units 159 and 57 maintained the time course of their response in background noise. MGTs for unit 159 were $3 \mathrm{~ms}$ and $2 \mathrm{~ms}$ in quiet and 18 $\mathrm{dB}$ S/N, respectively. MGTs for unit 57 were $2 \mathrm{~ms}$ in quiet and $5 \mathrm{~ms}$ in $12 \mathrm{~dB}$ $\mathrm{S} / \mathrm{N}$. Note that both TI units exhibit decreased spike counts outside of the gap stimulus in background noise, indicating that the background noise, presented alone, is inhibitory to these units. Both TE units are also driven by the background noise presented alone, as evidenced by elevated spike counts in noise both before and after the gap stimulus was presented. 
stimulus (Fig. 6). Recent studies have used artificially increased spontaneous neural activity to better visualize the time course of inhibition in auditory neurons (Bauer et al. 2000; Klug et al. 1999; Yang and Pollak 1998). In the IC, inhibition evoked by input to either ear could be more than twice as long as the stimulus duration (Bauer et al. 2000; Klug et al. 1999) and responses that outlasted the stimulus by $10-50 \mathrm{~ms}$ were routinely observed in the dorsal nucleus of the lateral lemniscus (Yang and Pollak 1998). Similar to our results above, the duration of inhibition in these studies was intensity dependent, with louder stimuli eliciting longer periods of inhibition. Together, these results suggest that tonic inhibitory responses that outlast the stimulus, and a concomitant poor temporal acuity, may be common for TI units throughout the auditory system and across species.
Decreased inhibitory duration and improved temporal acuity in background noise

The observed decrease in inhibitory duration produced by background noise might give rise to the improved temporal processing of TI units. A representation of how this might work is shown in Fig. 8. The inset displays the actual time course of inhibition to control stimuli measured in a representative TI unit. The $\mathrm{S} / \mathrm{N}$ ratio is shown along the $y$-axis with high $\mathrm{S} / \mathrm{N}$ ratios (low background noise levels) at the top of the graph. Time is plotted along the $x$-axis and the times at the onset and offset of inhibition at each $\mathrm{S} / \mathrm{N}$ ratio are shown by vertical lines. The inhibitory response demonstrated a systematic decrease in duration with increasing background noise level (decreasing $\mathrm{S} / \mathrm{N}$ ratio). This was produced by both a longer latency to onset of inhibition and a shorter latency to the offset of inhibition.

\section{Time Course of Inhibition}

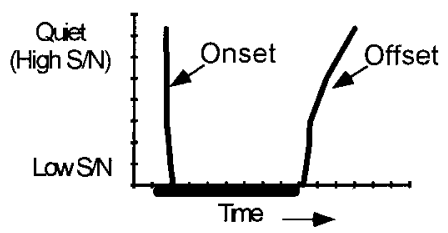

\section{Calculated Gap Responses}
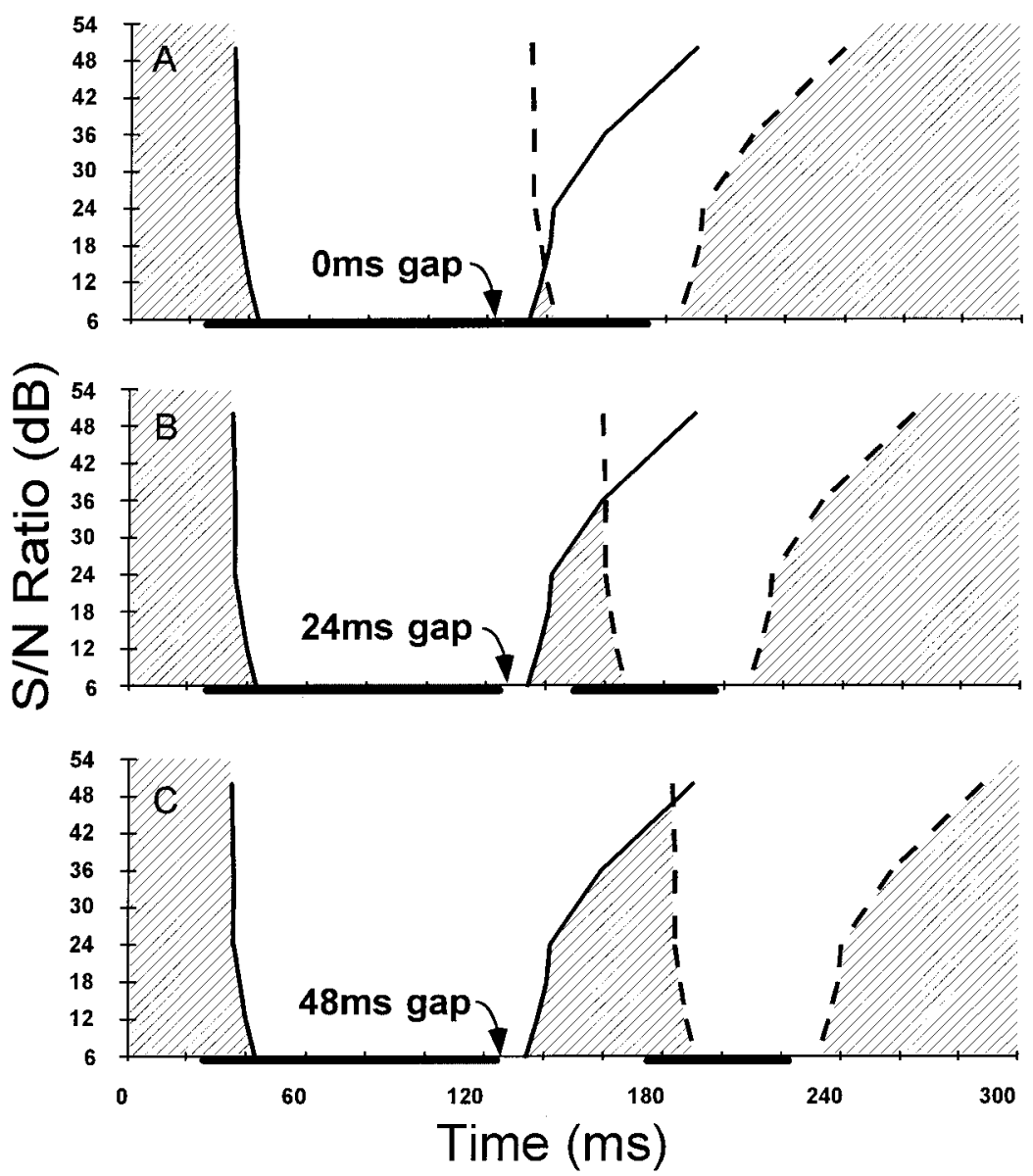

FIG. 8. Schematic diagram depicting the hypothetical time course of inhibition that underlies the effects observed in TI units. It is based on the time course of inhibition in the control response for a TI unit at various $\mathrm{S} / \mathrm{N}$ levels (inset). Onset and offset times were determined from control PST histograms as the first of 4 consecutive bins with counts below (for onset) or above (for offset) $30 \%$ of the maximum spike count in the control response at each background noise level. The ordinate represents the $\mathrm{S} / \mathrm{N}$ condition and the abscissa represents the temporal parameters of the gap stimulus (bars). In $A$, the hypothetical neural response to the control stimulus is shown, followed by the response to 24 - and 48 -ms gaps ( $B$ and $C$ ). The period of active inhibition is denoted by the unshaded areas. The shaded areas show the time and $\mathrm{S} / \mathrm{N}$ levels at which the spontaneous activity would be unaffected. 
We have applied these latencies, obtained for the entire 150-ms control stimulus, to a hypothetical 100-ms NB1 and 50-ms NB2, separated by gaps of different durations (Fig. 8, $A-C)$. In the hypothetical responses to the three gap durations, the shaded areas represent time/intensity combinations outside of the inhibitory response, where spontaneous activity would be unaffected. For example, if NB2 followed NB1 by a 24-ms silent gap (Fig. 8B), the inhibitory responses to NB1 and NB2 should overlap in low background noise levels $(>36 \mathrm{~dB} \mathrm{~S} / \mathrm{N}$, top of graph). This would prevent spontaneous activity from bleeding through the gap. However, for those same two noise bursts presented in higher background noise $(<36 \mathrm{~dB} \mathrm{~S} / \mathrm{N}$, bottom of graph), the truncated inhibitory responses would allow bleed through of spontaneous activity during the gap and decrease the MGT in poor S/N conditions.

There are minor differences between the predictions of this model and the observed response. This is expected due to differences in the temporal summation to the longer control stimulus than NB1 or NB2, different rise/fall times for the gap than for NB1 and NB2, and the influence of forward masking on the response to NB2. However, the model generally explains the improved MGTs in TI units in background noise and it appears likely that changing inhibitory response duration produces paradoxical gap detection thresholds in noise.

\section{Possible mechanisms decreasing inhibitory response duration}

A likely mechanism for shorter inhibitory response duration to NB1 and NB2 in background noise is neural adaptation. In excitatory responses, adaptation is thought to alter both the magnitude and the time course of neural response to acoustic stimuli (Chimento and Schreiner 1990, 1991; Feng et al. 1994; Harris and Dallos 1979; Kiang et al. 1965; Phillips 1990). In this study, tonically inhibited units also showed signs of adaptation in their response patterns. For many TI units, raising the background noise caused an increase in the spike count during the gap stimulus (e.g., Fig. 4, Unit 84 in Fig. 7), indicating less effective inhibition. Since the intensity of the gap carrier was $65 \mathrm{~dB}$ SPL regardless of background noise level, this suggests that factors outside the gap stimulus diminish the inhibitory efficacy of the foreground stimulus to the cell. Adaptation produced by the background noise is a likely source.

Other investigators have previously reported adaptation in auditory-evoked inhibitory responses. Finlayson and Adam (1997) demonstrated short-term adaptation in the inhibitory responses of EI neurons, where the inhibitory efficacy decreased during 200-ms tone bursts to the inhibitory ear. Moreover, intracellular recordings in cat auditory cortex have revealed decreasing inhibitory postsynaptic potential (IPSP) magnitude, indicative of inhibitory adaptation, during acoustically evoked inhibition (Volkov and Galazjuk 1991). Our findings reveal a novel consequence of inhibitory adaptation: paradoxical gap detection thresholds in background noise due to adaptation of the strong inhibitory input to TI units.

\section{Possible functional consequences}

In a population code of gap duration, the recruitment of TI units to shorter gap durations would increase the population of active neurons at a given gap duration and might then make the stimulus gap more detectable to the central target of such a code. However, psychophysical thresholds for gap detection closely parallel the temporal acuity of the auditory neurons which possess the shortest minimal gap thresholds (Klump and Gleich 1991; Klump and Maier 1989; Walton et al. 1997; Zhang et al. 1990). Moreover, even with the improvement brought about by background noise, the MGTs of TI units remain longer than those for TE and other units with excitatory response patterns. Thus, the large improvement in TI unit gap detection thresholds we observed would not necessarily translate into a large contribution to the perception of stimulus gaps. However, these findings do reveal an interesting change in the duration of inhibitory response, strength of inhibitory response, and resulting temporal acuity of TI units that may play a role in other signal processing contexts.

For example, both duration-tuned (Casseday et al. 1994; Ehrlich et al. 1997) and delay-tuned neurons (O'Neill and Suga 1979; Park and Pollak 1993; Saitoh and Suga 1995; Suga 1990) are believed to utilize long-lasting inhibitory input to extract stimulus features. Since inhibition is thought to set up delay lines for coincidence detection, manipulating stimulus level or $\mathrm{S} / \mathrm{N}$ ratio should change inhibitory duration and influence their tuning. In support of this hypothesis, Dear et al. (1993) found that decreasing the intensity of the first of two sounds shortens the delay tuning in delay-tuned cortical neurons. This would be expected based on the relationship between intensity and inhibitory duration observed in this and other studies (Bauer et al. 2000; Klug et al. 1999; Yang and Pollak 1998). We suggest here that shorter inhibitory duration, created by adding background noise, might also shorten the best duration or best delay of these cell types and play an important role in extracting shorter stimulus features under noisy conditions.

We thank M. Armour for histological processing, L. Moss for software development, and W. O'Neill for comments on earlier drafts of the manuscript.

This study was supported by National Institute on Aging Grant P01 AG09524.

\section{REFERENCES}

BARSZ K, BEnSON PK, AND WALton JP. Gap encoding by inferior collicular neurons is altered by minimal changes in signal envelope. Hear Res 115: 13-26, 1998.

Bauer E, Klug A, And Pollak G. Features of contralaterally evoked inhibition in the inferior colliculus. Hear Res 141: 80-96, 2000.

Buchfellner E, Leppelsack H-J, Klump G, and Hausler U. Gap detection in the starling (Sturnus vulgaris). II. Coding of gaps by forebrain neurons. J Comp Physiol [A] 164: 539-549, 1989.

CAsseday JH, Ehrlich D, AND COVEy E. Neural tuning for sound duration: role of inhibitory mechanisms in the inferior colliculus. Science 264: 847850, 1994.

Chimento TC And Schreiner CE. Time course of adaptation and recovery from adaptation in the cat auditory-nerve neurophonic. J Acoust Soc Am 88: 857-864, 1990.

Chimento TC and Schreiner CE. Adaptation and recovery from adaptation in single fiber responses in the cat auditory nerve. J Acoust Soc Am 90: 263-273, 1991.

Dear SP, Fritz J, Haresign T, Ferragamo M, and Simmons JA. Tonotopic and functional organization in the auditory cortex of the big brown bat, Eptesicus fuscus. J Neurophysiol 70: 1988-2009, 1993.

Dubno JR, Dirks DD, AND Morgan DE. Effects of age and mild hearing loss on speech recognition in noise. J Acoust Soc Am 76: 87-96, 1984.

ERlich D, CASSEDAy JH, AND COVEY E. Neural tuning to sound duration in the inferior colliculus of the big brown bat, Eptesicus fuscus. J Neurophysiol 77: 2360-2372, 1997. 
FAINGOLD CL, BOERSMA-ANDERSON CA, AND CASPARY DM. Involvement of GABA in acoustically-evoked inhibition in inferior colliculus neurons. Hear Res 52: 201-216, 1991.

FENG AS, LIN W-Y, AND Sun S. Detection of gaps in sinusoids by frog auditory nerve fibers: importance in AM coding. J Comp Physiol [A] 175: 531-546, 1994.

FINLAYSON PG AND ADAM TJ. Excitatory and inhibitory response adaptation in the superior olive complex affects binaural acoustic processing. Hear Res 103: 1-18, 1997.

FitzGibBons PJ. Temporal gap detection in noise as a function of frequency, bandwidth and level. J Acoust Soc Am 74: 67-72, 1983.

FitzGibBons PJ AND WightMAN FL. Gap detection in normal and hearingimpaired listeners. J Acoust Soc Am 72: 761-765, 1982.

ForREST TG AND GREEN DM. Detection of partially filled gaps in noise and the temporal modulation function. J Acoust Soc Am 82: 1933-1943, 1987.

FUZESSERY ZM AND HALL JC. Role of GABA in shaping frequency tuning and creating FM sweep selectivity in the inferior colliculus. J Neurophysiol 76: 1059-1073, 1996.

Giraudi D, Salvi RJ, Henderson DM, and Hamernik RP. Gap detection by the chinchilla. J Acoust Soc Am 68: 802-806, 1980.

Glasberg B AND MoOre B. Psychoacoustic abilities of subjects with unilateral and bilateral cochlear hearing impairments and their relationship to the ability to understand speech. Scand Audiol Suppl 32: 1-32, 1988.

Glasberg BR, MoORE BCJ, AND BACON SP. Gap detection and masking in hearing-impaired and normal-hearing subjects. J Acoust Soc Am 81: 15461556, 1987.

GoRdon-SALANT S AND FitzGibBons PJ. Temporal factors and speech recognition performance in young and elderly listeners. J Speech Hear Res 36: 1276-1285, 1993.

GREEN DM AND ForRest TG. Temporal gaps in noise and sinusoids. $J$ Acoust Soc Am 86: 961-970, 1989.

HARRIS DM AND DALlos P. Forward masking of auditory nerve fiber responses. J Neurophysiol 42: 1083-1107, 1979.

Ison JR, Bowen GP, Barlow JA, Taylor M, and Walton JP. Diminished response to acoustic transients in aged rodents. J Acoust Soc Am 93: 2409, 1993.

KIANG NYS. Discharge patterns of single fibers in the cats auditory nerve. MIT Monograph No. 35 105-112, 1965.

Klug A, Bauer EE, AND PollaK GD. Multiple components of ipsilaterally evoked inhibition in the inferior colliculus. J Neurophysiol 82: 593-610, 1999.

KLumP GM AND Gleich O. Gap detection in the European starling (Sturnus vugaris). III. Processing in the peripheral auditory system. J Comp Physiol [A] 168: 469-476, 1991.

KLUMP GM AND MaIER EH. Gap detection in the starling (Sturnus vulgaris). I. Psychophysical thresholds. J Comp Physiol [A] 164: 531-539, 1989.

Kuwada S, Batra R, Yin TCT, Oliver DL, Haberly LB, and Stanford TR. Intracellular recordings in response to monaural and binaural stimulation of neurons in the inferior colliculus of the cat. J Neurosci 17: 7565-7581, 1997.

Li L AND Kelly JB. Inhibitory influences of the dorsal nucleus of the lateral lemniscus on binaural responses in the rat's inferior colliculus. $J$ Neurosci 12: $4530-4539,1992$.

Mesulam MM. Tracing Neural Connections with Horseradish Peroxidase. New York: John Wiley, 1982.
OKANOYA K AND Dooling RJ. Detection of gaps in noise by budgerigars (Melopsittacus undulatus) and zebra finches (Peohila guttata). Hear Res 50: 185-192, 1990.

O'Neill WE and Suga N. Target range-sensitive neurons in the auditory cortex of the mustache bat. Science 203: 69-73, 1979.

PALOMBI PS AND CASPARY DM. GABA inputs control discharge rate primarily within frequency receptive fields of inferior colliculus neurons. J Neurophysiol 75: 2211-2219, 1996.

PARK TJ AND POLLAK GD. GABA shapes a topographic organization of response latency in the mustached bat's inferior colliculus. J Neurosci 13: 5172-5187, 1993.

PARK TJ AND POLLAK GD. Azimuthal receptive fields are shaped by GABAergic inhibition in the inferior colliculus of the mustache bat. $J$ Neurophysiol 72: 1080-1102, 1994.

PHILLIPS DP. Neural representation of sound amplitude in the auditory cortex: effects of noise masking. Behav Brain Res 37: 197-214, 1990.

Plomp R. Rate of decay of auditory sensation. J Acoust Soc Am 36: 277-284, 1964.

RoSEN S. Temporal information in speech acoustic, auditory and linguistic aspects. Philos Trans R Soc Lond B Biol Sci 336: 367-373, 1992.

SAITOH I AND SUgA N. Long delay lines for ranging are created by inhibition in the inferior colliculus of the mustached bat. J Neurophysiol 74: 1-11, 1995.

Shaller MJ AND Moore BC. Gap detection as a function of frequency, bandwidth, and level. J Acoust Soc Am 74: 467-473, 1983.

Stuart A AND PhilliPs DP. Word recognition in continuous and interrupted broadband noise by young normal-hearing, older normal-hearing, and presbyacusic listeners. Ear Hear 17: 478-489, 1996.

SugA N. Cortical computational maps for auditory imaging. Neural Networks 3: 3-21, 1990

Tyler R, Summerfield Q, Wood E, And Fernandes N. Psychoacoustic and phonetic temporal processing in normal and hearing-impaired listeners. $J$ Acoust Soc Am 72: 740-747, 1982.

VolKov IO AND GaLAZJUK AV. Formation of spike response to sound tones in cat auditory cortex neurons: interaction of excitatory and inhibitory effects. Neuroscience 43: 307-321, 1991.

Walton JP, Frisina RD, IsOn JR, and O'NeILl WE. Neural correlates of behavioral gap detection in the inferior colliculus of the young CBA mouse. J Comp Physiol [A] 181: 161-176, 1997.

WALTON JP, FRISINA RD, AND O'NeILL WE. Age-related alteration in processing of temporal sound features in the auditory midbrain of the CBA mouse. J Neurosci 18: 2764-2776, 1998.

Willott JF, Parham K, AND Hunter KP. Response properties of inferior colliculus neurons in middle-aged C57BL/6J mice with presbycusis. Hear Res 37: 15-28, 1988.

WillotT JF AND URBAN GP. Response properties of neurons in nuclei of the mouse inferior colliculus. J Comp Physiol [A] 127: 175-184, 1978.

YANG L AND POLLAK GD. GABAergic circuits sharpen tuning curves and modify response properties in the mustache bat's inferior colliculus. $\mathrm{J} \mathrm{Neu}$ rophysiol 68: 1760-1774, 1992.

YANG L AND POLLAK GD. Features of ipsilaterally evoked inhibition in the dorsal nucleus of the lateral lemniscus. Hear Res 122: 125-141, 1998.

ZhANG W, SALVI RJ, AND SAUNDERS SS. Neural correlates of gap detection in auditory nerve fibers of the chinchilla. Hear Res 46: 181-200, 1990. 